ACCepted to to ApJ Letters on July 20, 2013

Preprint typeset using $\mathrm{L}_{\mathrm{T}} \mathrm{EX}$ style emulateapj v. 5/2/11

\title{
A DEEP CHANDRA X-RAY LIMIT ON THE PUTATIVE IMBH IN OMEGA CENTAURI
}

\author{
Daryl Haggard, ${ }^{1}$ Adrienne M. Cool,${ }^{2}$ Craig O. Heinke, ${ }^{3}$ Roeland van der Marel ${ }^{4}$ Haldan N. Cohn, ${ }^{5}$ Phyllis M. \\ LUGGer, ${ }^{5}$ AND JAY ANDERSON ${ }^{4}$ \\ Accepted to to ApJ Letters on July 20, 2013
}

\begin{abstract}
We report a sensitive X-ray search for the proposed intermediate mass black hole (IMBH) in the massive Galactic cluster, $\omega$ Centauri (NGC 5139). Combining Chandra X-ray Observatory data from Cycles 1 and 13, we obtain a deep $(\sim 291 \mathrm{ks})$ exposure of the central regions of the cluster. We find no evidence for an X-ray point source near any of the cluster's proposed dynamical centers, and place an upper limit on the X-ray flux from a central source of $f_{\mathrm{X}}(0.5-7.0 \mathrm{keV}) \leq 5.0 \times 10^{-16} \mathrm{erg} \mathrm{cm}^{-2} \mathrm{~s}^{-1}$, after correcting for absorption. This corresponds to an unabsorbed X-ray luminosity of $L_{\mathrm{X}}(0.5-7.0$ $\mathrm{keV}) \leq 1.6 \times 10^{30} \mathrm{erg} \mathrm{s}^{-1}$, for a cluster distance of $5.2 \mathrm{kpc}$, Galactic column density $\mathrm{N}_{\mathrm{H}}=1.2 \times 10^{21}$ $\mathrm{cm}^{-2}$, and powerlaw spectrum with $\Gamma=2.3$. If a $\sim 10^{4} \mathcal{M}_{\odot}$ IMBH resides in the cluster's core, as suggested by some stellar dynamical studies, its Eddington luminosity would be $L_{\text {Edd }} \sim 10^{42} \mathrm{erg} \mathrm{s}^{-1}$. The new X-ray limit would then establish an Eddington ratio of $L_{\mathrm{X}} / L_{\mathrm{Edd}} \lesssim 10^{-12}$, a factor of $\sim 10$ lower than even the quiescent state of our Galaxy's notoriously inefficient supermassive black hole Sgr $A^{*}$, and imply accretion efficiencies as low as $\eta \lesssim 10^{-6}-10^{-8}$. This study leaves open three possibilities: either $\omega$ Cen does not harbor an IMBH or, if an IMBH does exist, it must experience very little or very inefficient accretion.

Subject headings: accretion, accretion disks — black hole physics — globular clusters: individual (NGC $5139)$
\end{abstract}

\section{INTRODUCTION}

Do globular clusters (GCs) harbor intermediate-mass black holes (IMBHs; $\left.\sim 10^{2}-10^{6} \mathcal{M}_{\odot}\right)$ ? Despite nearly four decades of study (e.g., Bahcall \& Ostriker 1975), this fundamental question in black hole astrophysics remains unanswered. IMBHs making up $\sim 0.1-1 \%$ of a cluster's mass could have formed at early times from runaway mergers of massive stars (Portegies Zwart et al. 2004), or from the evolution of population III stars (Madau \& Rees 2001). If they exist, these black holes would be of significant astrophysical interest, with potential connections to the assembly of supermassive black holes and to the first stars in the Universe (e.g., Volonteri 2012).

In the last decade, many GCs have been systematically searched for IMBHs. Dynamical measures of stars in the central regions have been undertaken to reveal the IMBH's influence, e.g., via high velocity dispersions near the sphere of influence (e.g., Gebhardt et al. 2000, and references therein) and have resulted in the identification of several candidates. Yet, these measurements are complicated both by competing physical mechanisms, e.g., mass segregation of populations

\footnotetext{
${ }^{1}$ CIERA Fellow, Center for Interdisciplinary Exploration and Research in Astrophysics, Physics and Astronomy Department, Northwestern University, 2145 Sheridan Road, Evanston, IL 60208, USA; dhaggard@northwestern.edu

2 Department of Physics and Astronomy, San Francisco State University, 1600 Holloway Ave., San Francisco, CA 94132, USA; cool@sfsu.edu

${ }^{3}$ Department of Physics, University of Alberta, Room 238 CEB, Edmonton, AB T6G 2G7, Canada

${ }^{4}$ Space Telescope Science Institute, 3700 San Martin Drive, Baltimore, MD 21218, USA

${ }^{5}$ Department of Astronomy, Indiana University, 727 E. Third St., Bloomington, IN 47405, USA
}

of lower-mass compact objects (e.g., Illingworth \& King 1976; Baumgardt et al. 2003), and by limitations in the available data, e.g., shot noise due to small numbers of stars within the sphere of influence (e.g., van der Marel \& Anderson 2010, vdMA10 hereafter).

Searches for accretion signatures at X-ray and radio wavelengths provide a complementary approach. G1, arguably the most convincing GC IMBH candidate in the Local Group (Ulvestad et al. 2007), has recently been studied by Miller-Jones et al. (2012) with simultaneous $\mathrm{X}$-ray and radio observations. They find X-ray emission consistent with earlier observations, but no detectable radio continuum emission, and argue that previous radio detections arise from flaring activity from a black hole low-mass X-ray binary (LMXB). As part of a larger campaign with the Jansky Very Large Array (JVLA), Strader et al. (2012) studied the cores of M15, M19, and M22, but uncovered no point sources consistent with the clusters' centers. They place $3 \sigma$ upper limits on the IMBH masses of $360-980 \mathcal{M}_{\odot}$. Thus, no clear evidence for IMBHs in globular clusters exists, even as there is substantial support for IMBHs in small galaxies (e.g., Greene \& Ho 2007; Thornton et al. 2008).

As the largest globular cluster in the Milky Way $\left(3 \times 10^{6} \mathcal{M}_{\odot}\right.$, Meylan 2002), or the possible remnant of an accreted dwarf galaxy (Norris et al. 1996), $\omega$ Cen is a prime candidate to harbor an IMBH. Significant efforts have been undertaken to search for one. Noyola et al. (2008, 2010) have claimed the dynamical detection of a $\sim 40,000 \mathcal{M}_{\odot}$ IMBH based on Hubble Space Telescope $(H S T)$ data and integral field unit spectroscopy from Gemini and VLT. However, another study based on HST proper motions (vdMA10; Anderson \& van der Marel 2010, hereafter AvdM10), achieves dynamical fits that do not require a massive compact central object, though 

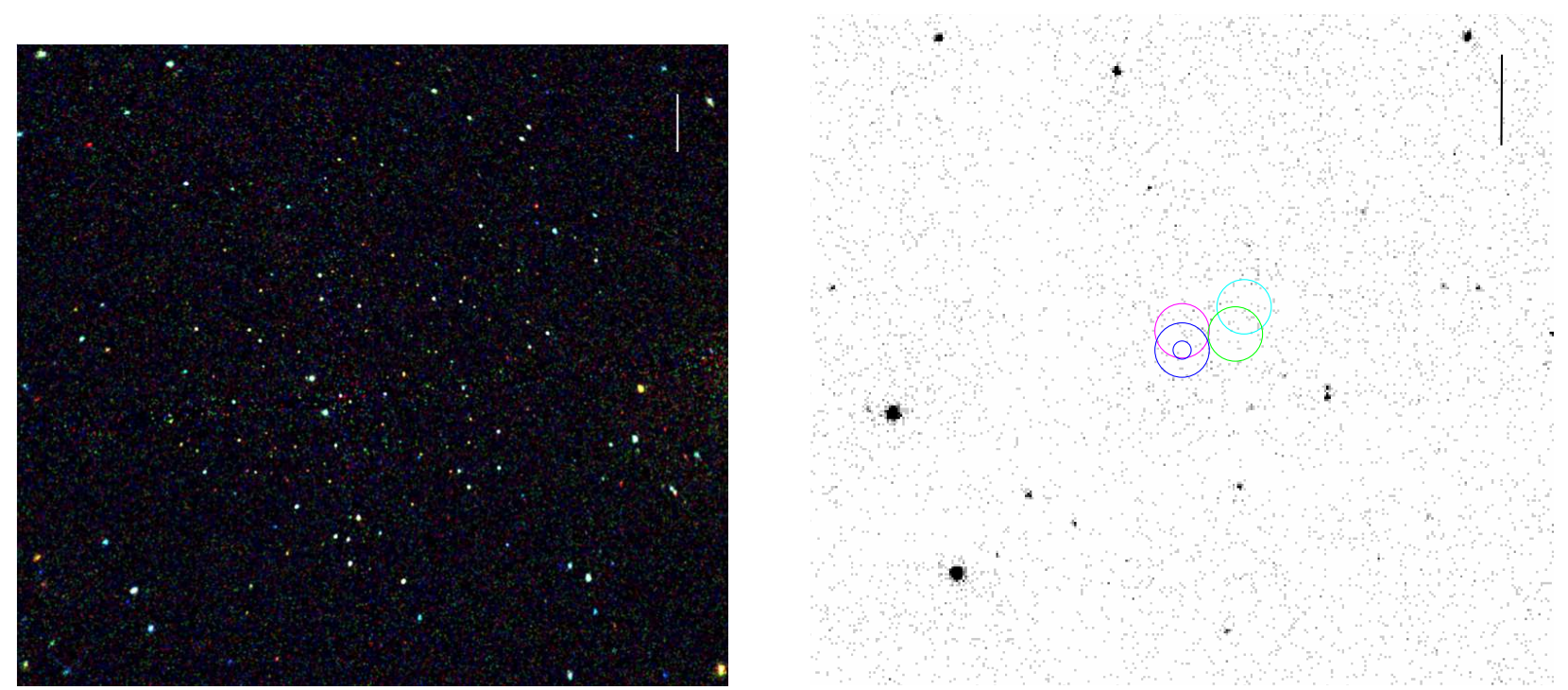

Figure 1. (Left) Chandra three-color X-ray image of the core of $\omega$ Cen (red: $0.5-1.2 \mathrm{keV}$, green: $1.2-2.0 \mathrm{keV}$, blue: $2.0-7.0 \mathrm{keV}$ ). The field of view is approximately $12^{\prime} \times 11^{\prime}\left(\sim 2.5\right.$ core radii $\left[\mathrm{r}_{c} \sim 2.6^{\prime}\right]$; white scale bar: $\left.1^{\prime}\right)$; North is up and East is to the left. The total combined Chandra exposure time is $290.9 \mathrm{ks}$. (Right) Broad band $\left(0.5-7.0 \mathrm{keV}\right.$ ) image of the inner $2.7 \times 2.5$ (black scale bar: $\left.20^{\prime \prime}\right)-$ no X-ray source is detected at or near any of the cluster's proposed centers (Table 1). The small (2"radius) blue circle marks the AvdM10 dynamical center; the large $6^{\prime \prime}$ radius circles indicate our search areas at the AvdM10 (blue), Novola et al. (2010) (magenta), Novola et al. (2008) (green), and Harris (1996) (cyan) centers. These represent the regions within which the putative IMBH may have "wandered" due to Brownian motion. The brightest X-ray sources are cataclysmic variables identified in HST images (Cool et al. 2013), allowing for accurate placement of the optical center on the image (see $\$ 2$ for details). (A color version of this figure is available in the online journal.)

their $1.8 \times 10^{4} \mathcal{M}_{\odot}(3 \sigma)$ upper limit still leaves room for a black hole in the IMBH mass range.

Most recently, Lu \& Kong (2011) have found a $3 \sigma$ peak in their $5.5 \mathrm{GHz}$ radio map of $\omega$ Cen at a position consistent with the center measured by AvdM10. Deeper radio imaging is required to determine if this peak is real, but the discovery of such peaks at the centers of both $\omega$ Cen and 47 Tuc is notable ( $\mathrm{Lu} \&$ Kong 2011).

ROSAT observations of $\omega$ Cen have revealed multiple sources in the cluster core, but none coincident with the cluster center (Verbunt \& Johnston 2000). Cycle 1 Chandra observations, sensitive to sources as faint as $L_{\mathrm{X}}(0.5-2.5 \mathrm{keV}) \approx 1 \times 10^{30} \mathrm{erg} \mathrm{s}^{-1}$ (Haggard et al. 2009 ), revealed many more sources in the core, but again nothing at the cluster center.

We have recently acquired Cycle 13 Chandra observations that increase the total exposure by a factor of $\gtrsim$ four. We present results of a more sensitive IMBH search carried out using the full Chandra data set. In Section 2, we describe the observations and our search for sources in and near the cluster center. In $\S 3$, we present new IMBH X-ray flux, luminosity, and mass limits, and discuss the implications of this non-detection; in $\S 4$ we summarize our findings.

\section{OBSERVATIONS AND ANALYSIS}

We obtained four Chandra exposures of $\omega$ Cen using the imaging array of the Advanced CCD Imaging Spectrometer (ACIS-I) in "very faint" mode, on 2000 January 24-25 (ObsIDs 653, 1519) and 2012 April 16-17 (ObsIDs 13726,13727$)$. We reduced and analyzed the data using CIAO6, reprocessing the observations and combining them using the chandra_repro and merge_obs scripts. The total combined on-axis exposure time is $290.9 \mathrm{ks}$. We used the known $H S T$ positions for the two brightest core sources (CVs 13a and 13c, Haggard et al. 2009; Cool et al. 2013) to perform a boresight correction $\left(\Delta \mathrm{RA}, \Delta \mathrm{Dec}=00^{\prime \prime} 0037,-0 \prime \prime 1130\right)$. The left panel of Figure 1 shows a smoothed X-ray three-color image of $\omega$ Cen; the right panel displays a broad-band $(0.5-7.0$ $\mathrm{keV})$ image of the inner $2.7 \times 2.5$ with proposed centers marked.

We applied CIAO's wavdetect algorithm to events with energies in the range $0.5-7.0 \mathrm{keV}$, and adopted a source significance threshold of $10^{-6}$, which gives $\sim 1$ false detection per $10^{6}$ pixels (Freeman et al. 2002), with wavelet (spatial) scales of 1-16 in intervals of 2 . No Xray point source is detected at or near any of the cluster's proposed centers; the closest X-ray detection lies $>10^{\prime \prime}$ from the AvdM10 center (Fig. 10.

In searching for an X-ray source associated with a possible IMBH, we allow that it might wander due to Brownian motion resulting from energy exchange with individual stars. The IMBH's "wander radius" is described by Chatterjee et al. (2002) and scales as $\left\langle\mathrm{x}^{2}\right\rangle=$ $2 / 9 \mathrm{r}_{c}^{2} \mathcal{M}_{\star} / \mathcal{M}_{\mathrm{BH}}$, where $\mathrm{x}$ is the one-dimensional RMS offset from the cluster center, $\mathrm{r}_{c}$ is the core radius, $\mathcal{M}_{\star}$ is the average stellar mass, and $\mathcal{M}_{\mathrm{BH}}$ is the mass of the IMBH. A less massive IMBH will experience larger per-

6 Chandra Interactive Analysis of Observations (CIAO) version 4.5 and Calibration DataBase (CALDB) version 4.5.5.1 (Fruscione et al. 2006). 
Table 1

$\omega$ Cen Reference Parameters \& X-ray Detection Limits

\begin{tabular}{|c|c|c|}
\hline Description & Best Value & Ref \\
\hline $\begin{array}{l}\text { Mass } \\
\text { Distance } \\
\text { Half-mass radius } \\
\mathrm{E}(\mathrm{B}-\mathrm{V}) \\
\text { Galactic } \mathrm{N}_{H}\end{array}$ & $\begin{array}{l}3 \times 10^{6} \mathcal{M}_{\odot} \\
5.2 \mathrm{kpc} \\
4.2^{\prime} \sim 6.3 \mathrm{pc} \\
0.11 \\
1.2 \times 10^{21} \mathrm{~cm}^{-2}\end{array}$ & $\begin{array}{l}1 \\
2 \\
2 \\
3 \\
4\end{array}$ \\
\hline $\begin{array}{l}\text { Proposed Centers } \\
\text { AvdM10 } \\
\text { Noyola }(2010)^{a} \\
\text { Noyola }(2008)^{b} \\
\text { Harris }(1996)\end{array}$ & $\begin{array}{l}\text { RA, Dec (J2000) } \\
13: 26: 47.24,-47: 28: 46.45 \\
13: 26: 47.24,-47: 28: 42.20 \\
13: 26: 46.08,-47: 28: 42.9 \\
13: 26: 45.9,-47: 28: 36.9\end{array}$ & $\begin{array}{l}5 \\
6 \\
7 \\
2\end{array}$ \\
\hline PL photon index $(\Gamma)$ & $\begin{array}{l}2.3(\mathrm{M} 15, \mathrm{M} 32) \\
2.7\left(\mathrm{Sgr} \mathrm{A}^{*}\right)\end{array}$ & $\begin{array}{l}8,9 \\
10\end{array}$ \\
\hline $\begin{array}{c}\text { X-ray detection limits } \\
f_{\mathrm{X}}(0.5-7.0 \mathrm{keV}) \\
L_{\mathrm{X}}(0.5-7.0 \mathrm{keV}) \\
f_{\mathrm{X}}(0.5-2.5 \mathrm{keV}) \\
L_{\mathrm{X}}(0.5-2.5 \mathrm{keV})\end{array}$ & $\begin{array}{l}(95 \% \text { confidence })^{c} \\
\leq 5.0 \times 10^{-16} \mathrm{erg} \mathrm{s}^{-1} \mathrm{~cm}^{-2} \\
\leq 1.6 \times 10^{30} \mathrm{erg} \mathrm{s}^{-1} \\
\leq 2.8 \times 10^{-16} \mathrm{erg} \mathrm{s}^{-1} \mathrm{~cm}^{-2} \\
\leq 9.0 \times 10^{29} \mathrm{erg} \mathrm{s}^{-1}\end{array}$ & $\begin{array}{l}11 \\
11 \\
11 \\
11\end{array}$ \\
\hline
\end{tabular}

Note. $-{ }^{a}$ The Novola et al. (2010) centroid has been tied to the master frame of AvdM10 at $(\mathrm{x}, \mathrm{y})=(6724,6895)$ and converted to RA, Dec using the 2 MASS point-source catalog. ${ }^{b}$ The Novola et al. (2008) centroid has been corrected by AvdM10 for HST guidestar errors. ${ }^{c}$ The total combined Chandra exposure time for these detection limits is $290.9 \mathrm{ks}$. The flux and luminosity limits are estimated for a PL with $\Gamma=2.3$, and corrected for Galactic absorption (see $\$ 2$ for details). Refs - 1: Mevlan (2002); 2: Harris (1996); 3: Lub (2002); 4: Willingale et al. (2013); 5: AvdM10; 6: Novola et al. (2010); 7: Noyola et al. (2008); 8: Ho et al. (2003a); 9: Ho et al. (2003b); 10: Baganoff et al. (2003); 11: This work.

turbations so we conservatively adopt $\mathcal{M}_{\mathrm{BH}}=1000 \mathcal{M}_{\odot}$ (among the lowest IMBH mass limits for $\omega \mathrm{Cen} 7$ ), and an average stellar mass of $0.8 \mathcal{M}_{\odot}$, which gives an RMS offset of $\sim 2^{\prime \prime}$. Hence, we search a circle with radius $\sim$ $6^{\prime \prime}(3 \sigma)$ at each of the possible cluster centers (Fig. 1).

We estimate the on-axis background in five large (45 pixel radius) source-free regions and find an average 0.5$7.0 \mathrm{keV}$ background level of 0.0643 counts/pixel. In these high quality Chandra data, on-axis sources are easily localized to within a 2-pixel radius $\left(\sim 1^{\prime \prime}\right)$, equivalent to an aperture area of $12.6 \mathrm{pix}^{2}$. With four possible cluster centers, each with a $6^{\prime \prime}$ search radius, we seek an IMBH in an area encompassing $~ 1800$ pixels. Given the very low background level, an on-axis source with just 6 counts in a $1^{\prime \prime}$ radius has a Poisson probability of $1.7 \times 10^{-4}$ and is likely to be real. Inspection of our search area confirms that none of the central regions contains a source with 6 or more counts.

We use the aprates tool with a $6^{\prime \prime}$ aperture at the AvdM10 cluster center to place an upper limit (95\% confidence) on the $0.5-7.0 \mathrm{keV}$ X-ray count rate of $4.43 \times 10^{-5}$ counts $\mathrm{s}^{-1}$. An IMBH accreting at a low fraction of its Eddington luminosity should be welldescribed by an absorbed power-law (PL), the spectral model used in numerous IMBH studies (e.g., Ho et al. 2003a, b; Miller-Jones et al. 2012), and in modeling lowluminosity active galactic nuclei (AGN, e.g., Dong et al.

7 This limit comes from the radio study of Lu \& Kong (2011), not a dynamical study, since Brownian motion would wash out the peak in the velocity dispersion that provides dynamical evidence for an IMBH.
2012). At a distance of $5.2 \mathrm{kpc}$ and assuming a PL spectrum with $\Gamma=2.3$ (Table 1), this detection limit corresponds to an unabsorbed flux limit of $f_{\mathrm{X}}(0.5-7.0 \mathrm{keV})$ $\leq 5.0 \times 10^{-16} \mathrm{erg} \mathrm{s}^{-1} \mathrm{~cm}^{-2}$, or a luminosity limit of $\leq 1.6 \times 10^{30} \mathrm{erg} \mathrm{s}^{-1}$ (see Table 1 for $0.5-2.5 \mathrm{keV}$ limits). Adopting the best fit PL for Sgr $\mathrm{A}^{*}$ in quiescence $(\Gamma=2.7$, Baganoff et al. 2003) yields a nearly identical result. This is the lowest $\mathrm{X}$-ray limit yet reported on an IMBH candidate in a globular cluster.

\section{RESULTS AND DISCUSSION}

\subsection{Eddington Ratio and Accretion Luminosity}

We convert this $L_{\mathrm{X}}$ limit to a bolometric luminosity limit via the standard correction $(\sim 7-20$, Elvis et al. 1994; Ho et al. 2003a), $L_{\text {bol }}<(1.1-3.2) \times 10^{31} \mathrm{erg} \mathrm{s}^{-1}$. The Eddington luminosity,

$$
L_{\text {Edd }}=1.26 \times 10^{38}\left(\mathcal{M}_{\mathrm{BH}} / \mathcal{M}_{\odot}\right) \mathrm{erg} \mathrm{s}^{-1}
$$

for the $3 \sigma$ upper limit on the IMBH mass from vdMA10 $\left(\leq 1.8 \times 10^{4} \mathcal{M}_{\odot}\right)$ is $L_{\text {Edd }}=2.3 \times 10^{42} \mathrm{erg} \mathrm{s}^{-1}$. This implies a very low Eddington accretion ratio of $L_{\mathrm{X}} / L_{\mathrm{Edd}}$ $<7.0 \times 10^{-13}$ or $L_{\text {bol }} / L_{\text {Edd }}<(5-14) \times 10^{-12}$.

For the simplest case of spherical accretion Bondi 1952), the accretion rate and luminosity (scaled to quantities appropriate for $\omega$ Cen) can be estimated from an optically thick, geometrically thin disk (Shakura \& Sunvaev 1973),

$$
\begin{aligned}
\dot{\mathcal{M}}_{\text {Bondi }} & =1.37 \times 10^{-8}\left(\frac{\mathcal{M}_{\mathrm{BH}}}{10^{4} \mathcal{M}_{\odot}}\right)^{2} \\
& \times\left(\frac{n}{0.038 \mathrm{~cm}^{-3}}\right)\left(\frac{T}{10^{4} \mathrm{~K}}\right)^{-3 / 2} \mathcal{M}_{\odot} \mathrm{yr}^{-1}, \text { and }
\end{aligned}
$$

$$
L_{\text {acc }, 1}=c^{2} \eta \dot{\mathcal{M}}_{\text {Bondi }},
$$

where $\eta$ is the accretion efficiency (Ho et al. 2003a). We estimate the core gas density following the formalism in Pfahl \& Rappaport (2001), based on free expansion of mass lost from stars, $n=$ $1\left(\frac{\mathcal{M}_{c}}{10^{5} \mathcal{M}_{\odot}}\right)\left(\frac{v_{w}}{20 \mathrm{~km} \mathrm{~s}^{-1}}\right)^{-1}\left(\frac{r_{\star}}{0.5 \mathrm{pc}}\right)^{-2} \mathrm{~cm}^{-3}$. We take the enclosed mass $\mathcal{M}_{\mathrm{c}}=\mathcal{M}_{\mathrm{h}}$ (i.e., half the cluster's mass), $r_{\star}=r_{\mathrm{h}}$ (the half-mass radius, $\sim 1.6 r_{\mathrm{c}}$ ), and a characteristic wind speed $v_{w}=50 \mathrm{~km} \mathrm{~s}^{-1}$, and find $n \sim 0.038$ $\mathrm{cm}^{-3}$, not unlike what Freire et al. (2001) measure for 47 Tuc. The gas temperature is assumed to be $T=10^{4} \mathrm{~K}$. For $\mathcal{M}_{\mathrm{BH}}=1.8 \times 10^{4} \mathcal{M}_{\odot}, L_{\mathrm{acc}, 1}=\eta 2.5 \times 10^{39} \mathrm{erg} \mathrm{s}^{-1}$. Comparing this accretion luminosity to the bolometric luminosity, we find $\eta \sim(4-13) \times 10^{-9}$. In this scenario, if $\omega$ Cen hosts such a massive IMBH, it must be a very inefficient accretor.

Maccarone \& Servillat (2008) have developed a more conservative approach for estimating IMBH accretion efficiencies and luminosities. They assume that (1) the accretion rate $(\dot{\mathcal{M}})$ is $\sim 3 \%$ of the Bondi rate for gas at $T=$ $10^{4} \mathrm{~K}$ (Pellegrini 2005); (2) the system is below the "lowhard state" transition (e.g., Maccarone 2003), wherein $\dot{\mathcal{M}} / \dot{\mathcal{M}}_{\text {Edd }}<0.02$ for $\dot{\mathcal{M}}_{\text {Edd }}=L_{\text {Edd }} /\left(0.1 c^{2}\right)$; and $(3) \eta$ scales linearly with accretion rate - to insure continuity 


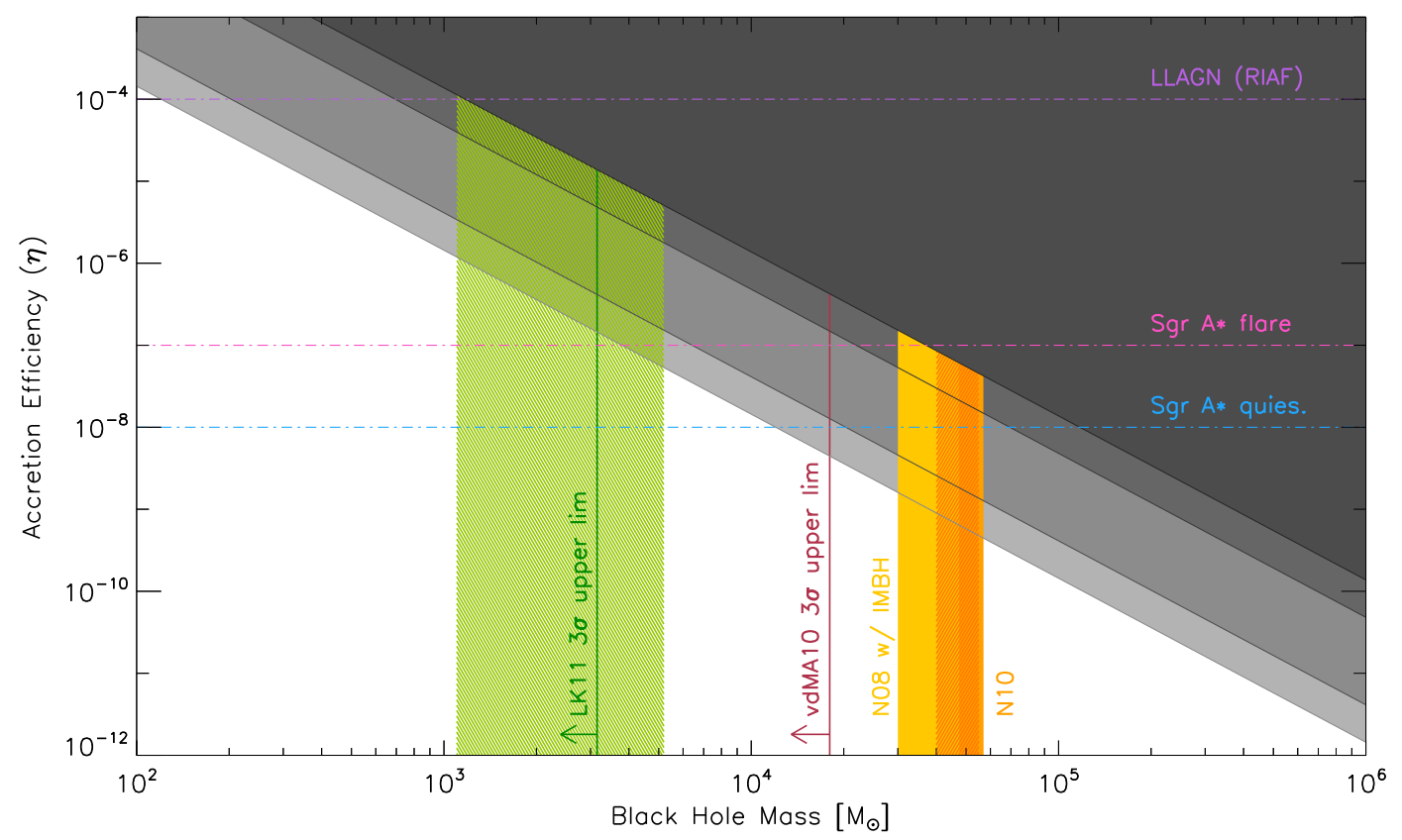

Figure 2. Exclusion plot for an accreting IMBH in $\omega$ Cen (mass of the black hole vs. accretion efficiency $[\eta]$ ) for our new X-ray upper limit, $L_{\mathrm{X}}(0.5-7.0 \mathrm{keV}) \leq 1.6 \times 10^{30} \mathrm{erg} \mathrm{s}^{-1}$. The light $\left(L_{\mathrm{bol}} / L_{\mathrm{X}}=7\right)$ and medium $\left(L_{\mathrm{bol}} / L_{\mathrm{X}}=20\right)$ grey regions are excluded for a simple Bondi accretion scenario (Eqns. $2 \& 3$ ). The medium-dark and dark grey regions are excluded for the modified accretion description from Maccarone \& Servillat (2008), discussed in 3.1 (Eqn. 4). The gas density and temperature are taken to be $n \sim 0.038 \mathrm{~cm}^{-3}$ and $T=10^{4}$ $\mathrm{K}$, respectively (3.1). The colored vertical lines/polygons correspond to IMBH mass estimates or upper limits (with errors) from radio and dynamical studies: $3 \sigma$ radio upper limit from ATCA (best fit: dark green line, error interval: light green polygon, Lu \& Kong 2011); $3 \sigma$ upper limit from vdMA10, including both core and cusp dynamical models (dark red line; see $\$ 3.2$; mass range from a combined $H S T$ and IFU dynamical study (yellow polygon, Novola et al. 2008); revised HST plus IFU study for new (light orange polygon) and old (hashed dark orange polygon) dynamical centers (Novola et al.|2010). Horizontal dot-dashed lines mark efficiencies for various accreting black holes (see also Table 2): low-luminosity AGN (LLAGN with $\eta \sim 10^{-4}$; purple); Sgr A* $\left(\mathcal{M}_{\mathrm{BH}}=4.1 \times 10^{6} \mathcal{M}_{\odot}\right)$, during a flare $($ magenta, Nowak et al. 2012; ; Neilsen et al. 2013) and in quiescence (blue, Baganoff et al. 2003). An AGN $\left(\mathcal{M}_{\mathrm{BH}}>10^{6} \mathcal{M}_{\odot}\right)$ with a "canonical" thin-disk efficiency $(\eta=0.1)$ would lie two decades above the top of this plot and is excluded for the entire IMBH mass range.

(A color version of this figure is available in the online journal.)

at the transition $(\sim 0.1), \eta=0.1\left(\left(\dot{\mathcal{M}} / \dot{\mathcal{M}}_{E d d}\right) / 0.02\right)$.

The accretion luminosity is then

$$
L_{\text {acc }, 2}=c^{2} 4.5 \times 10^{-3}\left(\dot{\mathcal{M}}_{\text {Bondi }}^{2} / \dot{\mathcal{M}}_{\text {Edd }}\right),
$$

or $L_{\mathrm{acc}, 2} \sim 1.3 \times 10^{33} \mathrm{erg} \mathrm{s}^{-1}$, where $\mathcal{M}_{\mathrm{BH}}=1.8 \times 10^{4}$ $\mathcal{M}_{\odot}, \dot{\mathcal{M}}_{\text {Edd }}=4.0 \times 10^{-4} \mathcal{M}_{\odot} \mathrm{yr}^{-1}$, and $\eta=1.7 \times 10^{-5}$.

\subsection{Constraints on an $I M B H$}

A simple test for the existence of a $\sim 1.8 \times 10^{4} \mathcal{M}_{\odot}$ $\mathrm{IMBH}$ in $\omega$ Cen assumes a standard thin-disk accretion efficiency $\eta \sim 0.1$; the resulting accretion luminosity is $\sim 2.5 \times 10^{38} \mathrm{erg} \mathrm{s}^{-1}$. No source this bright is present in $\omega$ Cen. The brightest X-ray sources are two known CVs $\left(L_{\mathrm{X}}=1.4-1.5 \times 10^{32} \mathrm{erg} \mathrm{s}^{-1}\right.$, Haggard et al. 2009; Cool et al. 2013), both of which have optical counterparts and $L_{\mathrm{X}}$ several orders of magnitude below this hypothetical accreting IMBH.

We can convert the $L_{\mathrm{X}}$ (or $L_{\text {bol }}$ ) upper limit to an IMBH mass upper limit by assuming a modeldependent accretion efficiency for a radiatively inefficient accretion flow (RIAF, e.g., Yuan et al. 2003; Naravan \& McClintock 2008, and references therein) with a low accretion efficiency $\left(\eta \sim 10^{-4}\right)$. For $L_{\text {acc, } 1}=$ $L_{\text {bol }}$, Eqns. 2 and 3 then imply $\mathcal{M}_{B H} \lesssim(120-200) \mathcal{M}_{\odot}$. The more conservative accretion scenario that informs
Eqn. 4 supports a similarly low accretion efficiency, and a comparison between $L_{\mathrm{acc}, 2}$ and $L_{\mathrm{bol}}$ implies $\mathcal{M}_{B H} \lesssim$ $(3.7-5.3) \times 10^{3} \mathcal{M}_{\odot}$. Even in the conservative case, this is an order of magnitude below dynamical estimates.

Figure 2 summarizes these findings as an exclusion plot based on the black hole mass and the accretion efficiency (Eqns. 1-4). Our 0.5-7.0 keV X-ray luminosity limit rules out the grey regions, which represent the scenarios described in $\S 3.1$ - those combinations of $\mathcal{M}_{\mathrm{BH}}$ and $\eta$ would result in an observable X-ray flux. IMBH mass limits (or expectations) from radio and dynamical studies are shown as colored polygons; efficiencies for several known massive black hole systems are indicated for reference. An $\mathrm{IMBH}$ in the expected range for $\omega$ Cen $\left(3 \times 10^{3}-3 \times 10^{4} \mathcal{M}_{\odot}\right)$ must be accreting with very low efficiency $\left(\eta<10^{-5}-10^{-9}\right)$ to fall below this low X-ray limit. A scenario in which $\omega$ Cen does not host an IMBH cannot be ruled out and, under most thin-disk and RIAF models, this scenario is preferred. The gas density and temperature of the accretion flow are based on theoretical predictions (§3.1) - a measure of the conditions in $\omega$ Cen's ambient medium would help to tighten these constraints.

\subsection{The Fundamental Plane}


Table 2

Black Holes Exhibiting Low-Efficiency Accretion

\begin{tabular}{lcccc}
\hline \hline Target & $\begin{array}{c}\mathcal{M}_{\mathrm{BH}} \\
\left(\mathcal{M}_{\odot}\right)\end{array}$ & $\begin{array}{c}L_{\mathrm{X}} \\
\left(\mathrm{erg} \mathrm{s}^{-1}\right)\end{array}$ & $L_{\mathrm{X}} / L_{\text {Edd }}$ & Ref. \\
\hline Proposed & GC IMBHs & & & \\
$\omega$ Cen & $<1.8 \times 10^{4}$ & $<1.6 \times 10^{30}$ & $<7.0 \times 10^{-13}$ & 1,2 \\
M54 & $\sim 9.4 \times 10^{3}$ & $<1.5 \times 10^{32}$ & $<1.4 \times 10^{-10}$ & 3,4 \\
M15 & $<2000$ & $<5.6 \times 10^{32}$ & $<2.2 \times 10^{-9}$ & 5,6 \\
\hline Known Accreting Black Holes \\
Sgr A*(q) & $4.1 \times 10^{6}$ & $\sim 2.4 \times 10^{33}$ & $5 \times 10^{-12}$ & 7 \\
Sgr A $A^{*}(\mathrm{f})$ & $4.1 \times 10^{6}$ & $(1-20) \times 10^{34}$ & $(2-40) \times 10^{-11}$ & $7,8,9$ \\
M32 & $2.5 \times 10^{6}$ & $9.4 \times 10^{35}$ & $3 \times 10^{-9}$ & 10 \\
\hline
\end{tabular}

Note. - For Sgr A* we include both (q: quiescent) and (f: flare) Xray properties. Refs - 1: This work; 2: vdMA10; 3: Ibata et al. (2009) 4: Wrobel et al. (2011); 5: Ho et al. (2003a); 6: Strader et al. (2012); 7 : Baganoff et al. (2003); 8: Nowak et al. (2012); 9: Neilsen et al. (2013); 10: Ho et al. (2003b).

The Fundamental Plane (FP) for black hole activity is an observed correlation between radio luminosity $\left(L_{\mathrm{R}}\right)$, $\mathrm{X}$-ray luminosity, and black hole mass (Merloni et al. 2003; Falcke et al. 2004), designed for estimating $M_{B H}$ for given $L_{\mathrm{R}}$ and $L_{\mathrm{X}}$. Miller-Jones et al. (2012) give a recent formulation of the FP in a study of the cluster G1 in M31,

$$
\begin{aligned}
& \log \mathcal{M}_{\mathrm{BH}}=(1.638 \pm 0.070) \log L_{\mathrm{R}} \\
& \quad-(1.136 \pm 0.077) \log L_{\mathrm{X}}-(6.863 \pm 0.790) .
\end{aligned}
$$

With only upper limits on $L_{\mathrm{X}}$ and $L_{\mathrm{R}}$ in $\omega$ Cen, application of the FP is not likely to be valid. If we conclude that there is no true radio detection, the FP provides no information. If instead we assume that the $2.5 \sigma$ radio detection from Lu \& Kong (2011, $f_{\mathrm{R}}=17.5 \mu \mathrm{Jy}$ at 5.5 $\mathrm{GHz})$ is real, we find $L_{\mathrm{R}} \sim 1.1 \times 10^{27} \mathrm{erg} \mathrm{s}^{-1}$. Combining this with our X-ray limit 8 would produce a lower limit on the IMBH mass of $\mathcal{M}_{B H} \gtrsim 1.2 \times 10^{3} \mathcal{M}_{\odot}$. This is in contrast to the IMBH mass upper limit derived for simple Bondi accretion (Eqns. 2 and 3), but not inconsistent with the mass upper limit estimated for the modified Bondi scenario (Eqn. 4). Hence, if the radio detection is real, the accretion source may fall on the FP. However, we conclude that the FP does not actually help constrain the mass of any IMBH in $\omega$ Cen, given the current observations.

\section{4. $\omega$ Cen in Context}

A similar dearth of IMBH accretion signatures has been noted in several other GCs, including M15, M19, and M22 (Strader et al. 2012), and the above-mentioned case of G1 (Miller-Jones et al. 2012). The dynamical measures that have led to claims for IMBHs (Ulvestad et al. 2007; Novola et al. 2008, 2010), as well as in NGC 6388 (Lützgendorf et al. 2011), and M54 (Ibata et al. 2009), remain controversial and may be in error (radio non-detections have also been reported for NGC 6388 and M54, Cseh et al. 2010; Wrobel et al. 2011), or simply suffer from large uncertainties. If the dynamical measures are not in error, we may be probing an unfamiliar accretion regime, in which the ambient gas is extremely sparse, or the accretion is very inefficient.

\footnotetext{
${ }^{8}$ Eqn. 5 requires $L_{\mathrm{X}}$ in the $0.5-10 \mathrm{keV}$ band; we convert our $0.5-7 \mathrm{keV}$ limit using PIMMS and the parameters in Tab. 1 to find $L_{\mathrm{X}}(0.5-10 \mathrm{keV})<1.8 \times 10^{30} \mathrm{erg} \mathrm{s}^{-1}$.
}

A handful of black holes are known to accrete with very low X-ray efficiencies; we include several examples in Table 2, For a massive IMBH in $\omega$ Cen $\left(1.8 \times 10^{4} \mathcal{M}_{\odot}\right)$, $L_{\mathrm{X}} / L_{\mathrm{Edd}}$ would be the lowest even in this poorly understood regime. Of course, the IMBH in $\omega$ Cen may be less massive or may not be present, leaving an alternate question: Why hasn't an IMBH formed at the dynamical center of this dense stellar system?

\section{SUMMARY}

We find no evidence for an X-ray source associated with $\omega$ Cen's cluster center in a deep $(\sim 291 \mathrm{ks})$ Chandra co-add. We report an X-ray upper limit for the (unabsorbed) flux and luminosity of $f_{\mathrm{X}}(0.5-7.0 \mathrm{keV})<$ $5.0 \times 10^{-16} \mathrm{erg} \mathrm{s}^{-1} \mathrm{~cm}^{-2}$, and $L_{\mathrm{X}}(0.5-7.0 \mathrm{keV})<$ $1.6 \times 10^{30} \mathrm{erg} \mathrm{s}^{-1}$, respectively. If an IMBH with $\mathcal{M}_{\mathrm{BH}} \sim 1.8 \times 10^{4} \mathcal{M}_{\odot}$ resides in $\omega$ Cen, it has the lowest Eddington ratio of any known massive $\mathrm{BH}$, even lower than Sgr A* in quiescence.

We appreciate discussions with Nicolas Cowan and Fred Rasio and thank the referee for helpful comments that improved the manuscript. $\mathrm{COH}$ is supported by an NSERC Discovery Grant and an Ingenuity New Faculty Award. This work is supported by Chandra Award Numbers GO2-13057A and GO2-13057B issued by the CXO, which is operated by the Smithsonian Astrophysical Observatory for and on behalf of NASA under contract NAS8-03060. This research made use of data obtained from the Chandra Data Archive and software provided by the Chandra X-ray Center (CXC) in the application packages CIAO, ChIPS, and Sherpa.

Facility: CXO (ACIS)

\section{REFERENCES}

Anderson, J., \& van der Marel, R. P. 2010, ApJ, 710, 1032

Baganoff, F. K., et al. 2003, ApJ, 591, 891

Bahcall, J. N., \& Ostriker, J. P. 1975, Nature, 256, 23

Baumgardt, H., Hut, P., Makino, J., McMillan, S., \& Portegies

Zwart, S. 2003, ApJ, 582, L21

Bondi, H. 1952, MNRAS, 112, 195

Chatterjee, P., Hernquist, L., \& Loeb, A. 2002, ApJ, 572, 371

Cool, A. M., Haggard, D., Arias, T., Brochmann, M., Dorfman, J., Gafford, A., White, V., \& Anderson, J. 2013, ApJ, 763, 126 Cseh, D., Kaaret, P., Corbel, S., Körding, E., Coriat, M.,

Tzioumis, A., \& Lanzoni, B. 2010, MNRAS, 406, 1049

Dong, R., Greene, J. E., \& Ho, L. C. 2012, ApJ, 761, 73

Elvis, M., et al. 1994, ApJS, 95, 1

Falcke, H., Körding, E., \& Markoff, S. 2004, A\&A, 414, 895

Freeman, P. E., Kashyap, V., Rosner, R., \& Lamb, D. Q. 2002, ApJS, 138, 185

Freire, P. C., Kramer, M., Lyne, A. G., Camilo, F., Manchester, R. N., \& D'Amico, N. 2001, ApJ, 557, L105

Fruscione, A., et al. 2006, in Society of Photo-Optical

Instrumentation Engineers (SPIE) Conference Series, Vol. 6270 Gebhardt, K., et al. 2000, ApJ, 539, L13

Greene, J. E., \& Ho, L. C. 2007, ApJ, 667, 131

Haggard, D., Cool, A. M., \& Davies, M. B. 2009, ApJ, 697, 224

Harris, W. E. 1996, AJ, 112, 1487

Ho, L. C., Terashima, Y., \& Okajima, T. 2003a, ApJ, 587, L35

Ho, L. C., Terashima, Y., \& Ulvestad, J. S. 2003b, ApJ, 589, 783

Ibata, R., et al. 2009, ApJ, 699, L169

Illingworth, G. D., \& King, I. R. 1976, PASP, 88, 607

Lu, T.-N., \& Kong, A. K. H. 2011, ApJ, 729, L25

Lub, J. 2002, in Astronomical Society of the Pacific Conference Series, Vol. 265, Omega Centauri, A Unique Window into Astrophysics, ed. F. van Leeuwen, J. D. Hughes, \& G. Piotto, 95 
Lützgendorf, N., Kissler-Patig, M., Noyola, E., Jalali, B., de Zeeuw, P. T., Gebhardt, K., \& Baumgardt, H. 2011, A\&A, 533, A36

Maccarone, T. J. 2003, A\&A, 409, 697

Maccarone, T. J., \& Servillat, M. 2008, MNRAS, 389, 379

Madau, P., \& Rees, M. J. 2001, ApJ, 551, L27

Merloni, A., Heinz, S., \& di Matteo, T. 2003, MNRAS, 345, 1057

Meylan, G. 2002, in Astronomical Society of the Pacific Conference Series, Vol. 265, Omega Centauri, A Unique Window into Astrophysics, ed. F. van Leeuwen, J. D. Hughes, \& G. Piotto, 3

Miller-Jones, J. C. A., et al. 2012, ApJ, 755, L1

Narayan, R., \& McClintock, J. E. 2008, New Astronomy Review, 51,733

Neilsen, J., et al. 2013, submitted to ApJ

Norris, J. E., Freeman, K. C., \& Mighell, K. J. 1996, ApJ, 462, 241

Nowak, M. A., et al. 2012, ApJ, 759, 95

Noyola, E., Gebhardt, K., \& Bergmann, M. 2008, ApJ, 676, 1008
Noyola, E., Gebhardt, K., Kissler-Patig, M., Lützgendorf, N., Jalali, B., de Zeeuw, P. T., \& Baumgardt, H. 2010, ApJ, 719, L60

Pellegrini, S. 2005, ApJ, 624, 155

Pfahl, E., \& Rappaport, S. 2001, ApJ, 550, 172

Portegies Zwart, S. F., Baumgardt, H., Hut, P., Makino, J., \& McMillan, S. L. W. 2004, Nature, 428, 724

Shakura, N. I., \& Sunyaev, R. A. 1973, A\&A, 24, 337

Strader, J., Chomiuk, L., Maccarone, T. J., Miller-Jones, J. C. A., Seth, A. C., Heinke, C. O., \& Sivakoff, G. R. 2012, ApJ, 750, L27

Thornton, C. E., Barth, A. J., Ho, L. C., Rutledge, R. E., \& Greene, J. E. 2008, ApJ, 686, 892

Ulvestad, J. S., Greene, J. E., \& Ho, L. C. 2007, ApJ, 661, L151

van der Marel, R. P., \& Anderson, J. 2010, ApJ, 710, 1063

Verbunt, F., \& Johnston, H. M. 2000, A\&A, 358, 910

Volonteri, M. 2012, Science, 337, 544

Willingale, R., Starling, R. L. C., Beardmore, A. P., Tanvir, N. R., \& O'Brien, P. T. 2013, MNRAS, 431, 394

Wrobel, J. M., Greene, J. E., \& Ho, L. C. 2011, AJ, 142, 113

Yuan, F., Quataert, E., \& Narayan, R. 2003, ApJ, 598, 301 